\title{
Titre de docteur honoris causa à Werner Bauer
}

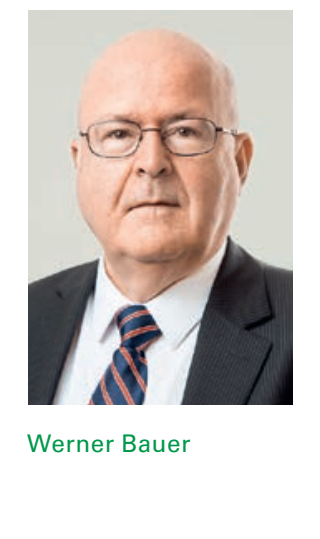

\section{Formation médicale marquée de son empreinte}

Lors du Dies academicus de cette année, j'ai eu l'immense plaisir de remettre virtuellement un titre honorifique à Werner Bauer et de prononcer les mots suivants: «La Faculté de médecine de l’Université de Zurich attribue le titre de docteur honoris causa au Dr Werner Bauer en reconnaissance de sa contribution essentielle à la formation postgraduée des médecins spécialistes, à leur formation continue tout au long de leur carrière et à l'insertion de cette formation dans l'enseignement universitaire. Le Dr Bauer a marqué de son empreinte la formation médicale en Suisse et au-delà des frontières; il a su lui insuffler des valeurs académiques en conciliant les intérêts de tous.» Par cette distinction, l'Université reconnaît qu'au-delà d'une recherche d'exception, une bonne formation médicale dans toutes ses phases et tout au long de la vie contribue de manière décisive à la qualité du système de santé et aux progrès de la médecine. Werner Bauer a mis tout son cœur à créer des structures de formation et à rechercher des synergies entre les acteurs de la formation médicale, pour que la prochaine génération de médecins puisse développer les compétences techniques, le professionnalisme et les hautes valeurs éthiques de la profession. Cher Werner, nous t'en remercions du fond du cœur!

Prof. Dr méd. Rainer Weber, doyen de la Faculté de médecine de l'Université de Zurich

\section{La FMH te remercie!}

La Faculté de médecine de l'Université de Zurich t’a décerné, cher Werner, le titre de docteur honoris causa en reconnaissance de ta contribution essentielle à la formation postgraduée des médecins spécialistes, à leur formation continue tout au long de leur vie et à l'insertion de cette formation dans l'enseignement universitaire. La FMH est fière de toi! En tant que président de l'Institut suisse pour la formation médicale postgraduée et continue, tu tiens les rênes de tout ce qui concerne la formation médicale postgraduée et continue au sein de la FMH, notre organisation médicale. Grâce à ton pragmatisme, tu as su faire fi d'un environnement politique et professionnel exigeant et obtenir des progrès significatifs dans le domaine de la formation postgraduée et continue, sans jamais perdre de vue les institutions académiques. Tu as fondamentalement amélioré la structure et la qualité de la formation postgraduée en Suisse. La FMH t'en remercie vivement! Au fond, tu veux nous rappeler: «Use your five senses. We miss more by not seeing than we do by not knowing» [1]. La FMH connaît la valeur de tes succès! La FMH admire plus particulièrement cet adage que tu t'es approprié: «But whatever you do, take neither yourselves nor your fellow-creatures too seriously» [2].

Dr méd. Jürg Schlup, président de la FMH

\section{Une source inestimable de connaissances au service du BMS}

Mon prédécesseur, Bruno Kesseli, avait demandé à Werner Bauer s'il accepterait de rejoindre la commission de rédaction du Bulletin des médecins suisses (BMS). C'était il y a treize ans. Il m’a aussi raconté qu'au début, il avait dû user de tout son talent de persuasion pour convaincre Werner Bauer d'assumer un rôle actif au sein du BMS. Nous nous estimons donc très heureux que Werner Bauer participe activement au BMS depuis plus d'une décennie. Les connaissances qu'il partage avec nous constituent une source de savoir inestimable en particulier pour statuer sur les articles que nous recevons. De manière constructive et posée, Werner Bauer nous aide à trouver une solution qui mettra tout le monde d'accord même dans les cas les plus délicats. Toute l'équipe des Editions médicales suisses (EMH) et du Bulletin des médecins suisses félicite chaleureusement Werner Bauer pour le titre de docteur honoris causa que lui a décerné l'Université de Zurich.

Dr Matthias Scholer, rédacteur en chef du Bulletin des médecins suisses 